\title{
The relative timing of trunk muscle activation is retained in response to unanticipated postural-perturbations during acute low back pain
}

\author{
S. Boudreau $\cdot$ D. Farina $\cdot$ L. Kongstad $\cdot$ \\ D. Buus $\cdot$ J. Redder $\cdot$ E. Sverrisdóttir . \\ D. Falla
}

Received: 5 October 2010/ Accepted: 7 March 2011/Published online: 26 March 2011

(c) The Author(s) 2011. This article is published with open access at Springerlink.com

\begin{abstract}
The purpose of this study was to assess the activation of the erector spinae (ES) and external oblique (EO) in response to unanticipated, bi-directional postural perturbations before and after the induction of acute low back pain (LBP) in healthy individuals. An experimental session consisted of a baseline, control, and an acute LBP condition. For the control and acute LBP condition, isotonic or hypertonic saline (HS), respectively, was injected into the right ES muscle. In each condition, participants stood on a moveable platform during which 32 randomized postural perturbations $(8$ repetitions of 4 perturbation types: $8 \mathrm{~cm}$ anterior slides, $8 \mathrm{~cm}$ posterior slides, $10^{\circ}$ anterior tilts, and $10^{\circ}$ posterior tilts) with varying interperturbation time intervals were performed over a period of 4-5 min. Bilateral surface electromyography (EMG) was recorded from the ES and $\mathrm{EO}$ in addition to subjective pain records. During the acute LBP condition: (1) the onset time of the ES and EO was delayed for the forward and backward sliding perturbations $(P<0.05)$; (2) EMG amplitude
\end{abstract}

S. Boudreau · L. Kongstad · D. Buus · J. Redder .

E. Sverrisdóttir

Center for Sensory-Motor Interaction,

Department of Health Science and Technology Faculty

of Medicine, Aalborg University, Aalborg, Denmark

D. Farina $\cdot$ D. Falla $(\bowtie)$

Department of Neurorehabilitation Engineering, Bernstein

Center for Computational Neuroscience, University Medical

Center Göttingen, Georg-August University, Von-Siebold-Str. 4,

37075 Göttingen, Germany

e-mail: deborah.falla@bccn.uni-goettingen.de

D. Falla

Pain Clinic, Center for Anesthesiology, Emergency and Intensive Care Medicine, University Hospital Göttingen, Robert-Koch-Str. 40, 37075 Göttingen, Germany was reduced bilaterally for all perturbations $(P<0.05)$; (3) the order of activation and interval between the onset times of the ES and EO were unaltered and (4) ES, but not EO, activity was adjusted to account for the directional differences between the perturbations. This study revealed that re-establishment of posture and balance was a result of the individuals' ability to rapidly modulate ES with respect to EO activity and that the bi-directional postural responses, although shifted in time and amplitude, retained temporal features in the presence of acute LBP.

Keywords Low back pain · Perturbation .

Motor control - Balance

\section{Introduction}

The ability to rapidly modulate the timing of muscles in response to unexpected postural perturbations is considered paramount for maintaining posture and balance. Automatic postural responses systematically vary with the direction of an external perturbation in both onset and amplitude (Moore et al. 1988; Rushmer et al. 1988; Jones et al. 2008). Additionally, gradual transitions between muscle onsets and amplitudes occur with fine changes in perturbation direction within the repertoire of these automatic postural responses (Moore et al. 1988). Alterations in somatosensation from the limbs or trunk, however, have been shown to modulate automatic postural responses. For example, individuals with peripheral neuropathy of the lower limbs show task-specific distal-to-proximal muscle activation strategies similar to controls following translations of the support surface, but exhibit delays at all segmental levels by $20-30 \mathrm{~ms}$ (Inglis et al. 1994). Additionally, these individuals show an inability to appropriately scale their 
postural responses to variations in perturbation velocity and amplitude. Although the slowed sensory conduction in the lower legs is likely to account for the delays, reduced somatosensation altered the ability to scale the magnitude of the postural response.

Individuals with chronic low back pain (LBP) also demonstrate delayed and reduced EMG activity compared with controls in response to anticipated (Hodges 2001) and unanticipated (Magnusson et al. 1996; Radebold et al. 2000) postural perturbations. Moreover, acute experimentally induced LBP in healthy individuals has been shown to alter trunk muscle activity during trunk flexion-extension (Zedka et al. 1999), as well as reaching (Thomas et al. 2007) and rapid-arm movement (Hodges et al. 2003) tasks. These findings indicate that individuals with acute or chronic LBP may have a biomechanical disadvantage and risk a fall or further injury in response to unexpected external perturbations.

Previous assessments of trunk muscle activity have employed protocols that approximate 'real-life' perturbations. This study examines, for the first time, automatic postural responses in the presence of experimentally induced LBP to fast, functionally relevant, full body perturbations. These perturbations are produced by a computer-controlled platform that can be controlled in threedimensional space to produce tilting or sliding perturbations that resemble tripping over an obstacle or slipping on a wet surface. The aim of the study was to determine if acute LBP, in healthy individuals, systematically alters the automatic postural responses to rapid tilting or sliding postural perturbations. Therefore, the onset time and amplitude of the EMG activity of the erector spinae (ES) and external oblique (EO) in response to anterior and posterior sliding and rotational tilting (toe-up and toe-down) of the support surface were compared before and after injection of hypertonic saline into the ES muscle. An examination of trunk muscle activity associated with functionally relevant postural perturbations, in healthy individuals, before and after experimentally induced LBP will reveal the immediate effects of pain on automatic postural responses.

\section{Methods}

\section{Participants}

Ten healthy individuals ( 4 men, age $23 \pm 1.05$ years, weight $69.8 \pm 13.52 \mathrm{~kg}$, height $171.8 \pm 9.31 \mathrm{~cm}$, BMI $23.45 \pm 2.73$ ) participated in a single experimental session. Individuals with previous or current respiratory, cardiovascular, or neurological conditions, low back pain or musculoskeletal disorders affecting the spine or limbs were excluded. Additionally, pregnant individuals or persons requiring prescription medication were excluded. All participants signed an informed consent form before the experimental session. Ethics approval for the study was granted by the regional Ethics Committee (No. 20090053) and the procedures were conducted according to the Declaration of Helsinski.

\section{Procedure overview}

The experimental session consisted of a baseline followed by a control and an acute LBP condition. The control condition consisted of an injection of isotonic saline (IS) and the acute LBP condition in an injection of the hypertonic saline (HS) into the right lower back. In the baseline condition or immediately after each injection, participants stood on a moveable platform during which 32 randomized postural-perturbations were performed. For each perturbation bilateral surface electromyography (EMG) was acquired from the ES and EO muscles.

Isotonic and hypertonic saline injections

A $1 \mathrm{ml}$ bolus of isotonic $(9 \mathrm{mg} / \mathrm{ml} \mathrm{NaCl}, \mathrm{SAD}$, Denmark) or hypertonic ( $1 \mathrm{mmol} / \mathrm{ml} \mathrm{NaCl}$, SAD, Denmark) saline was injected with a $0.4 \times 19 \mathrm{~mm}$ needle (BD Microlance $^{\mathrm{TM}} 3$, Becton-Dickinson, Ireland) into the right ES, $40 \mathrm{~mm}$ lateral to the spinous process at the second lumber vertebrae (Zedka et al. 1999; Hodges et al. 2003). The injection site was cleaned with alcohol prior to each injection. While the isotonic saline injection always preceded that of hypertonic saline, subjects were not aware which injection they were to receive. They were told that two would be given, and that one, both, or neither might be painful. Injections were given while the subject was in a prone position. Shortly $(<1 \mathrm{~min})$ after an injection, participants were assisted to a standing position and then onto the perturbation platform located directly in front of them.

Subjective pain intensity, quality and area

During the control and acute LBP conditions, the participants verbally conveyed their pain intensity to the investigator using a numerical rating scale (NRS) every $20 \mathrm{~s}$ for the duration of the 32 perturbations. Participants were asked to rate their pain on a scale from 0 to 10 , where 0 represented no pain and 10 represented the worst pain imaginable. Immediately following the cessation of the perturbations, participants completed a McGill Pain Questionnaire (MPQ) (Melzack 1975) and then shaded the area of perceived pain, if any, on a standardized body chart. Three or more occurrences of a pain quality descriptor from the MPQ were considered common and reported in the results. 
Postural-perturbations

Postural-perturbations were produced using a computercontrolled movable platform (Andersen and Sinkjaer 2003). Participants were instructed to step onto the centre of the platform and position their feet, approximately, shoulder-width apart such that they were able to stand in a comfortable posture. Four different postural-perturbations were produced with varying inter-perturbation time intervals (Range 7-20 s) in order to reduce the participants' ability to predict the type and perturbation onset. The four perturbations consisted of an $8-\mathrm{cm}$ anterior slide, $8-\mathrm{cm}$ posterior slide, 10 -anterior or posterior tilt of the platform around the middle axis in the frontal plane, refer to Fig. 1. The platform had a constant velocity of $270 \mathrm{~cm} / \mathrm{ms}$ and $120 \% \mathrm{~ms}$ from starting position (ground height and level) to the end position for the slides and tilts, respectively. Pilot trials revealed that these postural-perturbation parameters resulted in marked activity of the ES and EO muscles without producing discernable compensatory postural movements of the arms and legs. In each experimental condition, all four perturbations were repeated eight times in random order for a total of 32 perturbations. After each perturbation the platform slowly returned to the starting position. The 32 perturbations were completed over a 4- to 5-min interval and a 5-min rest period occurred between each experimental condition. Prior to the onset of the experimental protocol the participants experienced each type of postural-perturbation. These posturalperturbations were considered unanticipated since any specific anticipation to a perturbation direction or type would, conceivably, increase the risk of instability (Lavender et al. 1993).

\section{EMG recordings}

Bipolar surface EMG signals were detected from the ES and EO with pairs of electrodes $\left(\mathrm{Ambu}^{\circledR}\right.$ Neuroline 720 , Denmark) positioned $20 \mathrm{~mm}$ apart following skin preparation. Three pairs of electrodes were placed over the ES muscle bilaterally at $\mathrm{T} 12, \mathrm{~L} 2$, and $\mathrm{L} 4,40 \mathrm{~mm}$ lateral to the spinal column and one pair of electrodes was placed over EO muscle bilaterally, approximately $15 \mathrm{~cm}$ lateral to the umbilicus. An $\mathrm{Ag} / \mathrm{AgCl}$ self-adhesive reference electrode $\left(A m b u^{\circledR}\right.$ Neuroline Ground, Denmark) was attached over the spine at L2. The EMG signals were sampled at $2,048 \mathrm{~Hz}$ with a gain of 2,000 using a 128-channel surface EMG-USB amplifier (LISiN-OT Bioelettronica, Torino, Italy; $-3 \mathrm{~dB}$ bandwidth $10-500 \mathrm{~Hz}$ ) and converted to digital form by a 12-bit analog-to-digital converter.

A trigger signal originating from the computer-controlled platform was used as a reference in order to determine the onset of ES and EO activity following each perturbation. The average rectified value (ARV) was estimated from the EMG signals over non-overlapping signal epochs of $50 \mathrm{~ms}$. The first $50 \mathrm{~ms}$ epoch was recorded prior to the perturbation onset and the remaining epochs were recorded thereafter. The ARV of EMG was assessed as an average over $300 \mathrm{~ms}$ following the onset of the perturbation. The onset of muscle activity was determined using the signal processing method developed by Merlo and colleagues (Merlo et al. 2003).

\section{Statistical analysis}

A three-way repeated measures analysis of variance (ANOVA) was used to determine the main effects of condition (baseline, control, acute LBP) on EMG amplitude or onset for each muscle (ES, EO) and for each side (left, right). A two-way ANOVA was used to determine differences between EMG onset or amplitude for perturbation types (anterior slide, posterior slide, anterior tilt; posterior tilt) across conditions (baseline, control, acute LBP); the left and right side of each muscle was grouped. Significant differences revealed by ANOVA were followed by post hoc Student Newman-Keuls (SNK) test. Results are reported as mean and standard deviation (SD) in the text, and as standard error (SE) in figures. Statistical significance was set at $P<0.05$.
Fig. 1 Illustration of the four perturbation types with respect to the participant's stance

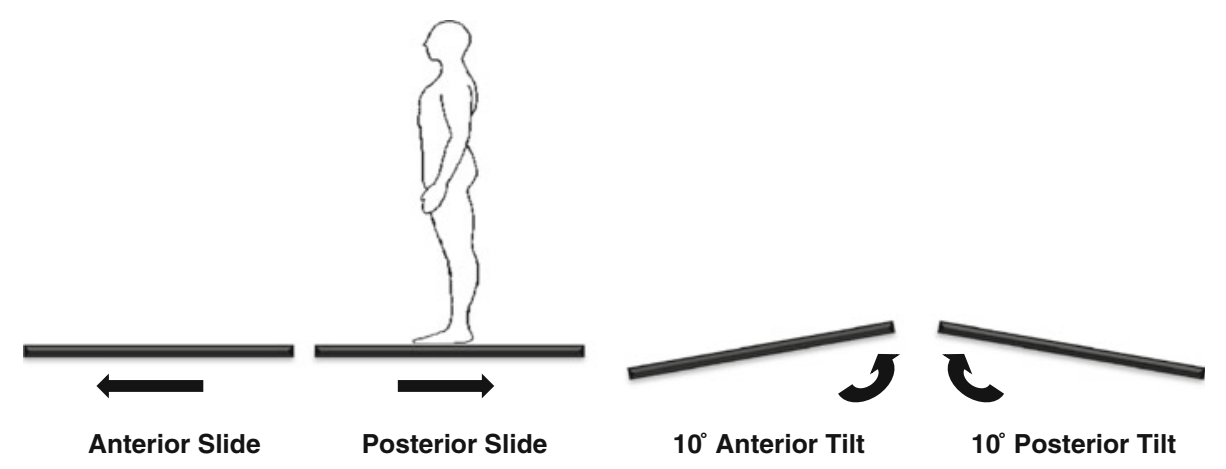




\section{Results}

Subjective pain intensity, quality and area

The mean pain intensity following injection of hypertonic saline was $3.9 \pm 0.5$. Peak pain intensity occurred at $80 \mathrm{~s}$ following injection of hypertonic saline and the pain intensity was greater than zero, for all participants, throughout the duration of the 32 perturbations, see Fig. 2. Participants described the quality of pain following the isotonic injection $(0.50 \pm 0.46)$ as boring, scalding, tender, annoying and tight. In contrast, participants described the quality of pain following the hypertonic injection as drilling, sharp, pressing, scalding, taut, miserable and tight, with scalding being the most common reported pain quality descriptor. The subjective shadings of pain area on the body chart, see Fig. 3, revealed that all participants

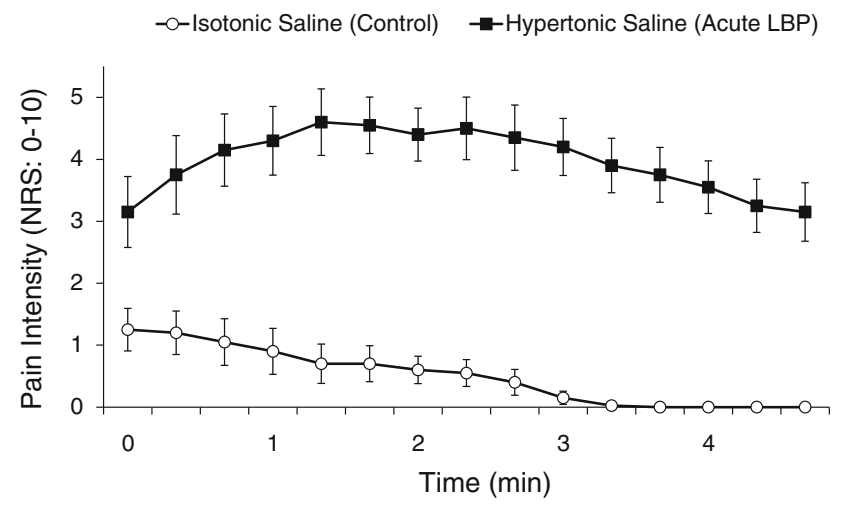

Fig. 2 Time course of subjective pain ratings associated with injections of isotonic or hypertonic saline into the right lower back perceived the pain following the hypertonic saline injection as being restricted to the side of injection (right side).

External oblique and erector spinae muscle activity before perturbations

The EMG amplitude of the EO or ES activity prior to each type of postural perturbation did not differ across conditions (ANOVA, $P>0.809$ ), nor was there a difference in the EMG amplitude between the left and right EO or ES (ANOVA, $P>0.15$ ).

Comparison of external oblique and erector spinae muscle activity within perturbations

All participants were able to regain posture and balance following the unanticipated postural-perturbations.

\section{Anterior slide}

For the acute experimental pain condition, there was a decrease in EMG amplitude (ANOVA, $F=6.7$; $P=0.006)$ for the ES and EO in comparison with the control (SNK, $P=0.013$ ) and baseline (SNK, $P=0.007$ ) conditions (Fig. 4a). As indicated in Fig. 4b, there was also a delay in EMG onset (ANOVA, $F=4.6 ; P=0.024$ ) for the ES and EO in comparison with the control (SNK, $P=0.036)$ and baseline (SNK, $P=0.020)$ conditions. There was, however, no difference between the left and right $\mathrm{ES}$ or $\mathrm{EO}$ across conditions for EMG amplitude (ANOVA, $F=1.7 ; P=0.20$ ) or EMG onset (ANOVA, $F=0.3 ; P=0.72$ ); data not shown. The EMG onset time for the ES was consistently later than the EO (ANOVA,
Fig. 3 Shadings of perceived area of pain associated with the injection of isotonic (a) or hypertonic (b) saline into the right lower back
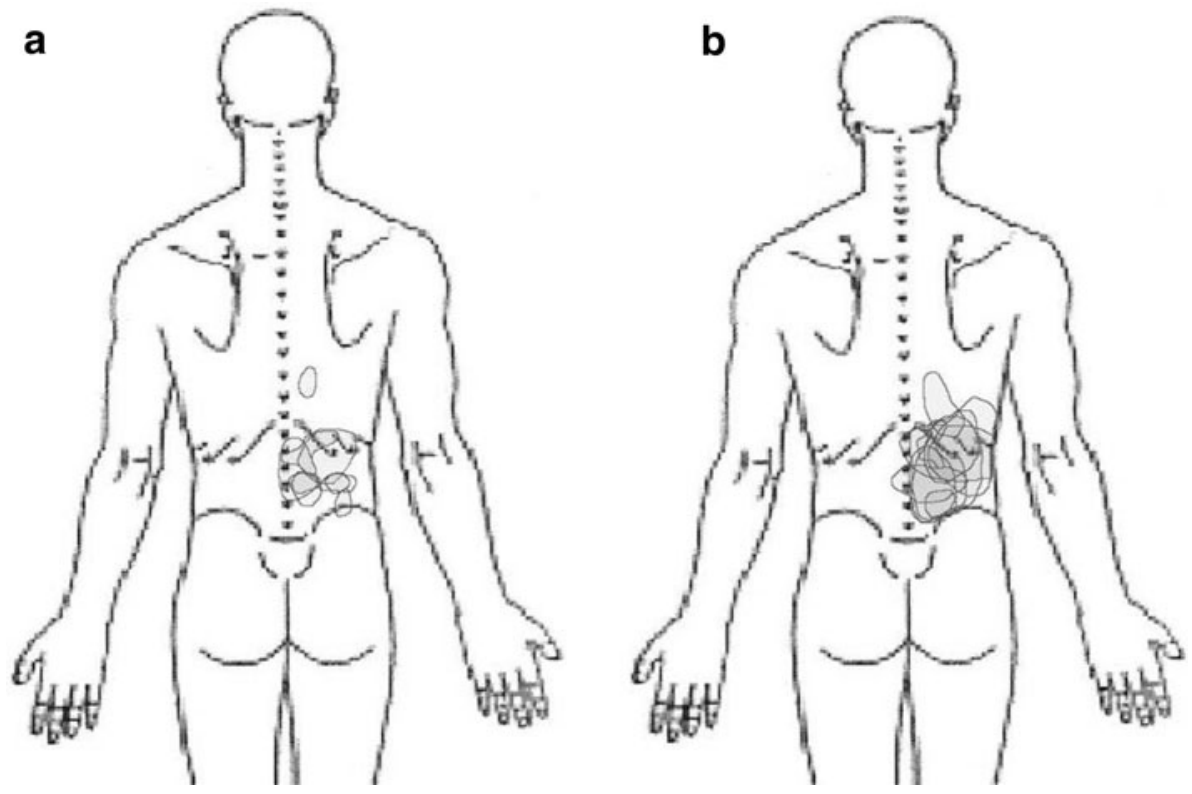
$F=29.519, P=0.0004$; SNK, $P=0.0005)$ and the difference between the ES and EO onset times was retained during acute LBP (ANOVA, $F=0.5, P=0.594$ ).

\section{Posterior slide}

For the acute experimental pain condition, there was a decrease in EMG amplitude (ANOVA, $F=4.1, P=$ 0.033 ) for the ES and EO in comparison with the control (SNK, $P=0.037$ ) and baseline (SNK, $P=0.04)$ conditions (Fig. 4b). There was also a tendency for a delay in EMG onset (ANOVA, $F=3.6, P=0.047$ ) for the pain condition in comparison with the control (SNK, $P=0.043)$ and baseline (SNK, $P=0.059)$ conditions (Fig. 4d). Consistent with the data for the anterior slide perturbation, there was no difference between the left and right $\mathrm{ES}$ or $\mathrm{EO}$ across conditions for EMG amplitude (ANOVA, $F=0.9, P=0.403$ ) or EMG onset (ANOVA, $F=0.2, P=0.77$ ); data not shown. The EMG onset time for the ES was consistently earlier than the EO (ANOVA, $F=5.9, P=0.037$; SNK, $P=0.037$ ) and the difference between the ES and EO onset times was retained during acute LBP (ANOVA, $F=0.1, P=0.896$ ).

\section{Anterior tilt}

For the acute experimental pain condition, there was a modest decrease in EMG amplitude (ANOVA, $P=0.040$ ) for the ES and $\mathrm{EO}$ in comparison with the baseline (amplitude: SNK, $P=0.036$ ), but not the control (SNK, $P=0.074$ ) condition (Fig. 5a). Furthermore, there was no change in EMG onset (ANOVA, $F=0.544 ; P=0.589$;
Fig. 5b), and similar to the anterior and posterior slides, there was no difference between the left and right ES or EO across conditions for EMG amplitude (ANOVA $F=0.4$, $P=0.636$ ) or onset times (ANOVA, $F=0.1 ; P=0.826$ ); data not shown. For this particular postural-perturbation the EMG onset times for the ES and EO were similar (ANOVA, $F=0.9, P=0.344$ ) and this similarity was retained for the acute LBP condition (ANOVA, $F=1.0$, $P=0.362)$.

\section{Posterior tilt}

There was a tendency for a decrease in EMG amplitude (ANOVA, $F=3.4 ; P=0.052$ ) for the ES and EO for the acute experimental pain condition in comparison with the control (SNK, $P=0.048$ ) and baseline (SNK, $P=0.068$ ) conditions (Fig. 5b). Similar to the anterior tilt, there was no change in EMG onset (ANOVA, $F=0.4 ; P=0.669$; Fig. 5d). Consistent with the slides and anterior tilt, there were no differences between the left and right ES or EO across conditions for EMG amplitude (ANOVA, $F=0.150 ; P=0.861$ ) or onset time (ANOVA, $F=0.4$; $P=0.669)$; data not shown. Nor was there a difference between the EMG onset times for the ES and EO (ANOVA, $F=0.02, P=0.877$ ) which is consistent with the acute LBP condition (ANOVA, $F=0.1, P=0.896$ ).

\section{Comparison of external oblique and erector spinae} muscle activity across perturbations

For the purpose of clarity and given the finding of no differences between the left and right EMG activity for the
Fig. 4 Mean of the left- and right EMG amplitude (a, b) and onset time $(\mathbf{c}, \mathbf{d})$ of the erector spinae (ES) and external oblique (EO) during the baseline, control, and low back pain conditions for the anterior and posterior sliding perturbations. *Represents significant post hoc SNK results $(P<0.05)$
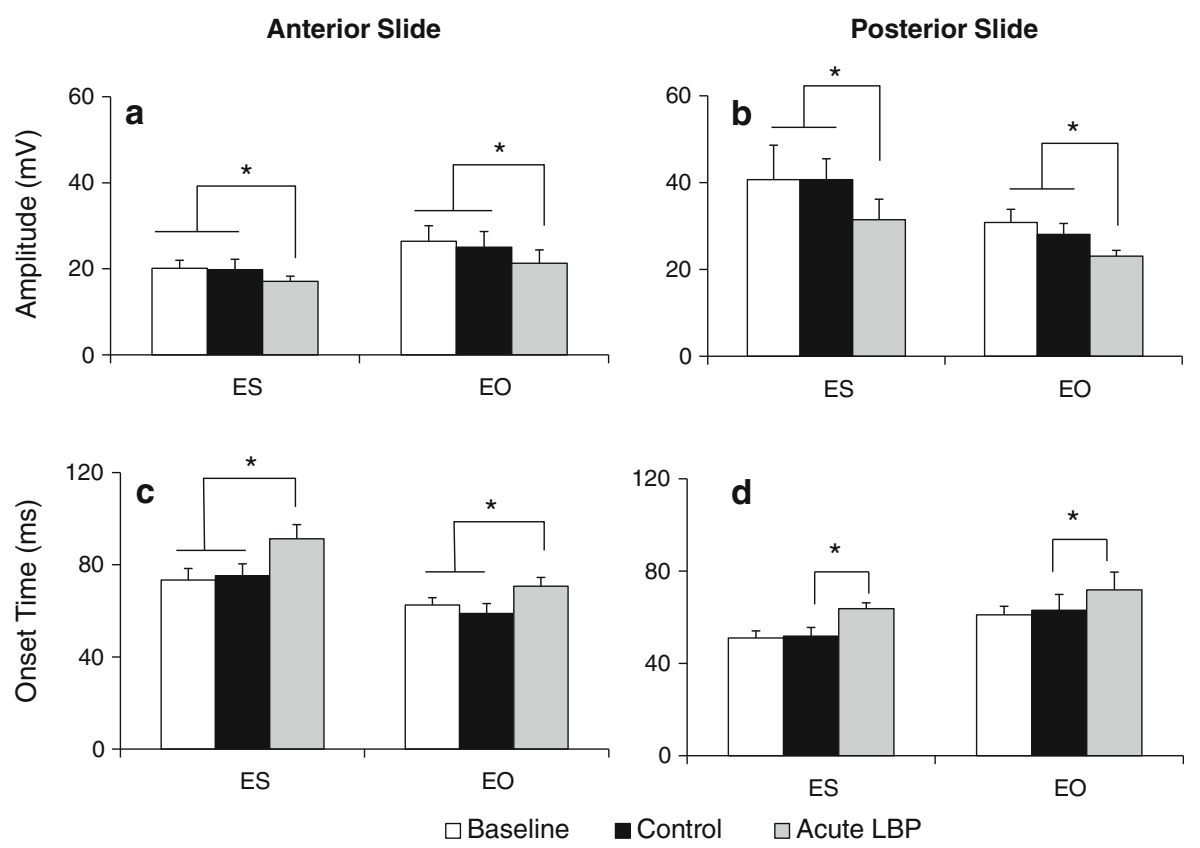
Fig. 5 Mean of the left and right EMG amplitude (a, b) and onset time $(\mathbf{c}, \mathbf{d})$ of the erector spinae (ES) and external oblique (EO) during the baseline, control and low back pain conditions for the anterior and posterior tilting perturbations. *Represents significant post hoc SNK results $(P<0.05)$
Anterior Tilt
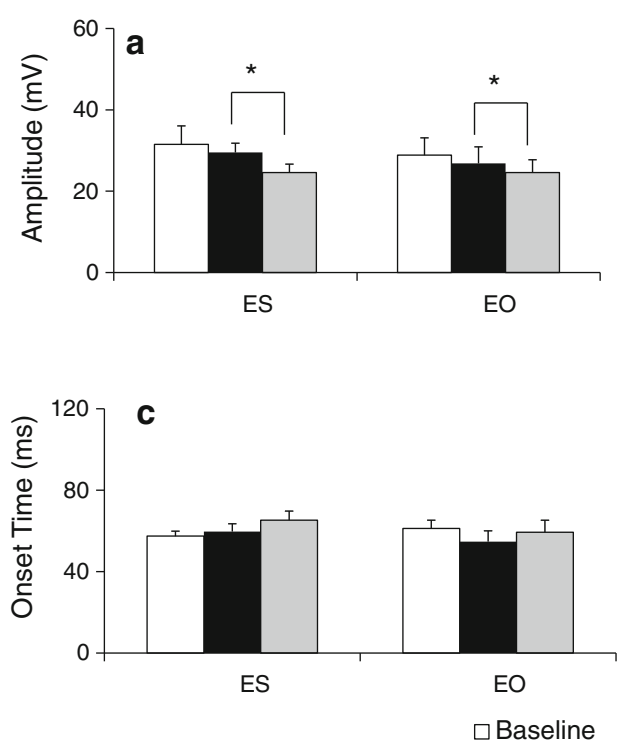

Posterior Tilt
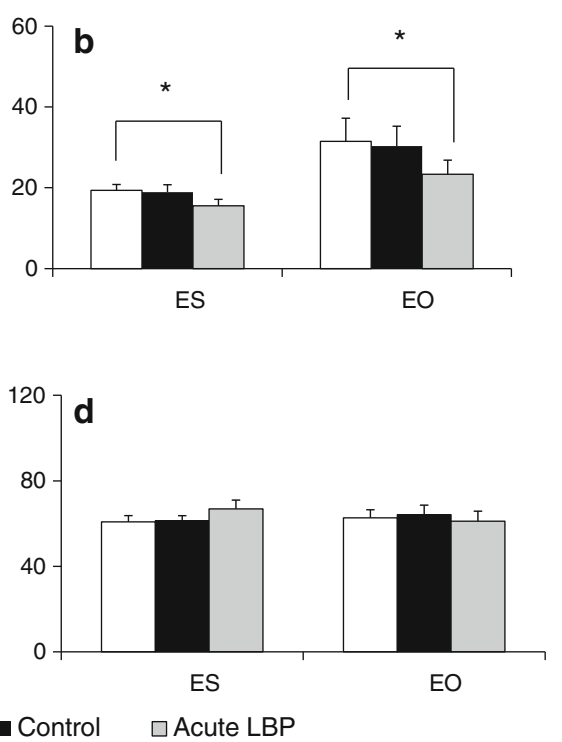

ES or EO, the left and right EMG activity data were pooled. Accounting for the main effect of condition on the activity of the EO and ES, namely a delay in onset time and/or a decrease in amplitude, further analyses revealed no differences between the amplitude (ANOVA, $F=0.8$, $P=0.494$ ) or onset time (ANOVA, $F=5.8, P=0.275$ ) of the EO between the four perturbation types. In contrast, each of the four perturbation types produced marked differences in the amplitude (ANOVA, $F=8.9, P=0.0003$ ) and/or onset time (ANOVA, $F=8.9, P=0.0003$ ) of the ES. These findings are summarized in Table 1 and the main effects of acute LBP on these predefined muscle activity patterns are expressed in Fig. 6a-d).

\section{Discussion}

There were three main findings in this study. First, acute unilateral experimental LBP resulted in a bilateral delay in EMG onset time and reduced EMG amplitude for the ES and EO, but only for the sliding perturbations. Second, the order of activation and the time interval between the onset of the ES and EO for the four perturbations were unaltered during the acute LBP condition. Third, only the onset time of the ES systematically varied with the differing perturbation types and directions. Together, these findings indicate that despite a delay in onset time and decreased EMG activity, the relative timing between the activation of the ES and EO was retained in response to rapid, unanticipated full-body perturbations during acute LBP.

In this present study, delays and decreased EMG activity of the ES and EO muscles were found following a unilateral injection of hypertonic saline in the low back in response to sliding postural perturbations. Previously, it has been shown that pre-activation of the trunk muscles, equivalent to $40 \%$ maximal extension effort, effectively reduced reflex responses of the trunk muscles to postural perturbations when compared with a $20 \%$ maximal extension effort (Stokes et al. 2000). However, given that the EMG activity of the EO and ES prior to the onset of all perturbation types (sliding and tilting) did not differ between conditions, the reduced EMG activity and delayed onsets of the EO and ES found in response to the sliding perturbations can be attributed to the acute LBP intervention.

Interestingly, the unilateral injection of hypertonic saline resulted in a unilateral perception of pain in the lower back but a bilateral delay and reduction in EMG activity of the ES and EO. A bilateral change in the recruitment strategy of ES and EO may have been necessary in order to maintain stability of the spine. If only the muscle activity of the ES and EO on the painful side was altered a torque moment would ensue following the postural perturbations, which would subject the spine to potentially damaging shearing forces. In contrast to limb muscles, for example, the left and right internal obliques have comparable representations in the ipsilateral and contralateral primary motor cortices (Strutton et al. 2004), which supports the possibility of bilateral motor control processes.

The marked decreases in the EMG activity and onset for the sliding perturbations, as opposed to the tilting perturbations may have resulted from differences in the overall displacement of the participant's centre-of-mass. For the sliding perturbations, inter-segmental moments of the spine would occur around the centre-of-mass, located within the trunk region, to a greater extent than for the tilting 
Table 1 Post hoc comparisons for perturbation type ( $P S$ posterior slide, $A S$ anterior slide, $P T$ posterior tilt, $A T$ anterior tilt) on the amplitude and onset times of the erector spinae (ES) muscle

\begin{tabular}{|c|c|c|c|c|c|c|c|c|}
\hline & \multicolumn{4}{|c|}{ Amplitude $(\mathrm{mV})^{\mathrm{a}}$} & \multicolumn{4}{|l|}{ Onset $(\mathrm{ms})^{\mathrm{a}}$} \\
\hline & PS & AS & $\mathrm{PT}$ & AT & PS & AS & PT & AT \\
\hline PS & & $<0.001$ & $<0.001$ & 0.0641 & & $<0.001$ & 0.0398 & 0.0793 \\
\hline AS & $<0.001$ & & 0.8355 & 0.0300 & $<0.001$ & & $<0.001$ & $<0.001$ \\
\hline PT & $<0.001$ & 0.8355 & & 0.0479 & 0.0398 & $<0.001$ & & 0.4531 \\
\hline AT & 0.0641 & 0.0300 & 0.0479 & & 0.0794 & $<0.001$ & 0.4531 & \\
\hline Mean (SD) & 40.67 (18.60) & $19.96(6.30)$ & $19.13(4.17)$ & 30.52 (9.67) & $51.43(9.19)$ & $74.29(10.24)$ & $61.10(6.06)$ & $57.37(4.86)$ \\
\hline
\end{tabular}

For clarity, a mean of the baseline and control amplitude and onset times has been listed. Bold value indicates significant difference

${ }^{a}$ Refer to Fig. 3 for the main effects of the acute low back pain condition on these results

Fig. 6 Mean of the left and right EMG amplitude (a, b) and onset $(\mathbf{c}, \mathbf{d})$ of the erector spinae (ES) and external oblique (EO) for each perturbation type ( $P S$ posterior slide, $A S$ anterior slide, $P T$ posterior tilt, $A T$ anterior tilt) for the mean of the baseline and control condition $(\mathbf{a}, \mathbf{b})$ and the acute low back pain (LBP) condition (c, d). Figures illustrate that balance was maintained from the adjustment of ES amplitude and onset latency for all perturbations type, in comparison with the relatively consistent behaviour of the EO for each perturbation type

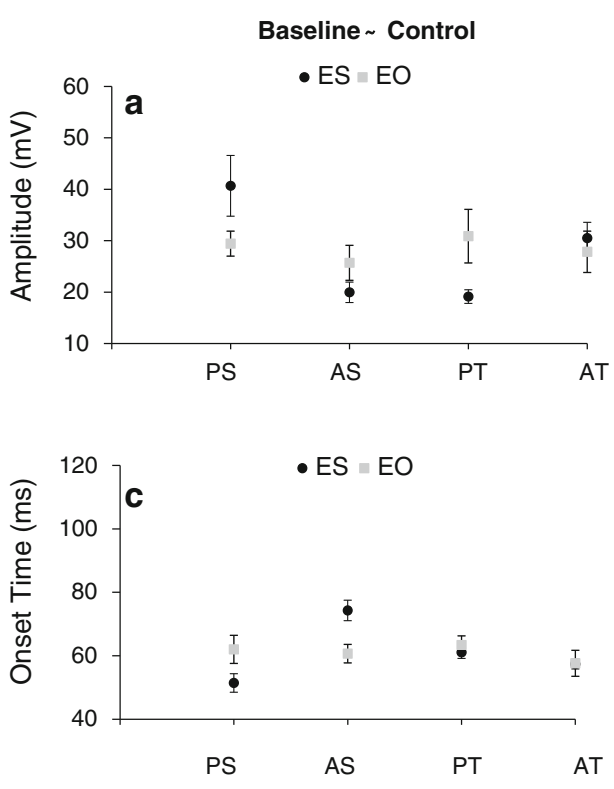

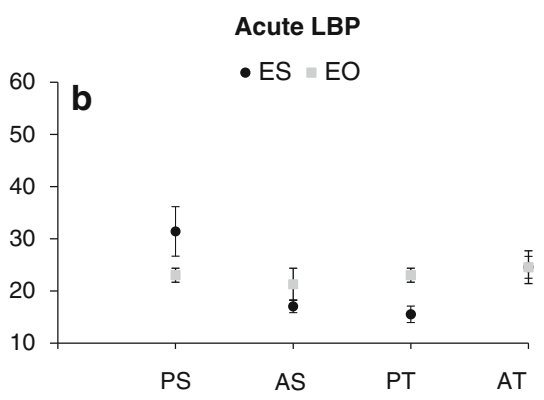

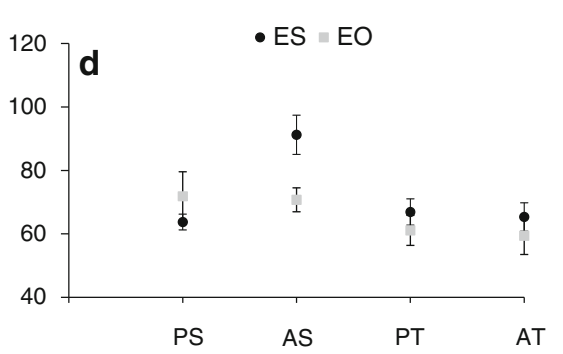

perturbations. In contrast, tilting would induce a greater stretch in the ankle and lower legs. Given the location of the painful intervention (low back) the onset time and EMG activity of the ES and EO is therefore more likely to be affected during the sliding, as opposed to the tilting perturbations. In accordance with this view, stretch reflexes elicited via the soleus or gastrocnemius may have triggered the postural response during the tilting perturbations (Bloem et al. 2000) as the ES muscles respond reflexively to activation of sensory receptors in the triceps surae muscles and Achilles tendon (Clair et al. 2009). Although EMG activity of the lower limb muscles were not specifically examined in the present study, it is likely that the postural response during the tilting perturbations was triggered by sensory cues distal to the trunk.

The delayed onsets and deceases in EMG activity of the ES and EO in the acute LBP condition also concur with reports of delays or decreased trunk muscle activity in the presence of pain during the performance of simple rudimentary tasks (Thomas et al. 2007) and following anticipated (Hodges 2001; Hodges et al. 2003) and unanticipated postural perturbations (Magnusson et al. 1996; Radebold et al. 2000). For example, deceased amplitude of the ES in response to sudden extension moments around the level of T4 has been shown during induced LBP in healthy individuals (Magnusson et al. 1996). Increased cocontraction has been suggested as a mechanism that would explain these decreases in amplitude since prior activation of the affected muscles reduces the amplitude of the needed response to off-set the perturbation (van Dieen et al. 2003). This suggestion, however, may only be applicable to anticipated postural perturbations since any specific anticipation, such as pre-tensioning of the ES muscles, would increase the risk of postural instability (Lavender et al. 1993).

In comparison with anticipated (anterior-posterior) postural perturbations, unanticipated perturbations can result in increased trunk displacement and muscle 
responses. However, when the temporal onset of the perturbation is well-known, there is a pre-activation of the trunk muscles and a reduction in trunk displacement and peak muscle activity following the perturbation (Thomas et al. 1998). Further, the rectus abdominus, TrA, EO and ES have been shown to become simultaneously activated following unanticipated perturbations (ventral loadings during standing) to the trunk (Cresswell et al. 1994). It has been suggested that activation of these trunk muscles following a postural perturbation serve to stabilize the spine by increasing intra-abdominal pressure. In this present study, a decrease in ES and EO muscle activity and delayed onsets were found during the acute LBP condition, which suggests that acute LBP may compromise the stability of the spine. It remains unclear at this time if the rectus abdominus and TrA, which largely generate intra-abdominal pressure, are similarly altered in response to unanticipated full body postural perturbations during LBP conditions.

The mechanisms contributing to the delayed muscle responses following postural perturbations in low back pain conditions remain unknown. Injection of hypertonic saline into the ES does not alter the stretch reflex in this muscle (Zedka et al. 1999), which suggests an involvement of supraspinal structures or central commands. Nonetheless, these present findings demonstrated that an acute LBP condition altered the automatic postural responses during the sliding perturbations, which means that an unexpected event, such as a slip on a wet surface, may place an individual with LBP at a greater risk of further injury.

Although the acute LBP condition was associated with delayed activity, the relative timing between the onset of ES and EO for all four postural perturbations was maintained. Comparable findings have been shown in individuals with peripheral neuropathy of the lower limbs. Despite a common delay in onset times, similar taskspecific distal-to-proximal muscle activation strategies were observed between these individuals and healthy controls in response to sliding perturbations (Inglis et al. 1994). Magnusson and colleagues (1990) estimated that adequate postural control can be maintained if somatosensory feedback delays do not exceed $300 \mathrm{~ms}$. This concurs with the findings of our study which showed that the delays (range 10-35 ms) did not lead to a failure in the re-establishment of posture. However, it is unknown if individuals with chronic LBP would exhibit the same relative timing between the onset of ES and EO during similar postural perturbations and whether adequate postural control could be maintained.

In the case of experimental or chronic LBP conditions, a shift from temporally decoupled to coupled muscle activity has also been demonstrated for the transverses abdominis (TrA) muscle during rapid arm-movements (Hodges and
Richardson 1996; Hodges 2001; Hodges et al. 2003). These studies have consistently shown that pre-recruitment of the TrA prior to the deltoid no longer occurs during the rapid arm-movement task. However, these studies investigated the response to anticipated perturbations, which means that feed-forward motor control strategies or a greater involvement of supraspinal structures (Jacobs and Horak 2007) was employed to re-establish posture. Individuals with chronic LBP also demonstrate decreased variability in anticipatory postural adjustments (Jacobs et al. 2009), which suggests a reduction in the repertoire of motor control strategies utilised to re-establish posture. In comparison with healthy individuals, LBP patients demonstrate the inability to switch off agonist and turn on antagonist muscles during unanticipated extension moments around the trunk (Radebold et al. 2000). Zedka and colleagues (1999) have also shown reduced ES activity during extension and increased ES activity during relaxation following hypertonic saline injection into the ES of healthy individuals. These studies suggest that acute or chronic LBP may compromise the ability to temporally decouple or modulate the onset times between trunk muscles which may increase the risk of fall or further injury.

Differing EMG onset and amplitudes of the ES and EO in response to the four unanticipated postural perturbations were found in this study. Opposing perturbation directions, namely the anterior- and posterior-related perturbations, produced distinct EMG onset and amplitudes of the ES and EO. As expected, the ES differed from the EO in both amplitude and onset for the posterior sliding perturbation and this difference was reversed for the anterior slides. An important note is that the EMG onset time of the ES occurred approximately $20 \mathrm{~ms}$ earlier for the posterior slide in comparison with the anterior slide perturbation. Although not as pronounced, this difference in ES onset time was also evident between the posterior and anterior tilting perturbations (approximately $10 \mathrm{~ms}$ ). However, further analysis revealed that unlike the ES, the onset of the EO did not systemically vary with the direction or the type of the postural perturbation. These results indicate that reestablishment of posture and balance was a result of the individuals' ability to rapidly and independently modulate ES with respect to EO activity. Moreover, this study showed that individual motor strategies (differences in the timing and amplitude of the ES and EO) for the anteriorposterior and posterior-anterior sliding and tilting perturbations (with and without pain) exist for healthy individuals. If the ability to independently modulate, relax or decouple muscle acitivity is compromised, as is evident in chronic LBP conditions (Radebold et al. 2000; Hodges et al. 2003), then the ability to safely re-establish posture and balance following an unexpected event will also be compromised. 


\section{Conclusion}

This study showed a delay in onset time and a decrease in the amplitude of EMG activity of the ES and EO in response to rapid, unanticipated full-body perturbations during an acute LBP condition in healthy individuals. Despite these changes, the order of activation and interval between the onset times of the ES and EO were unaltered.

Open Access This article is distributed under the terms of the Creative Commons Attribution Noncommercial License which permits any noncommercial use, distribution, and reproduction in any medium, provided the original author(s) and source are credited.

\section{References}

Andersen JB, Sinkjaer T (2003) Mobile ankle and knee perturbator. IEEE Trans Biomed Eng 50:1208-1211

Bloem BR, Allum JH, Carpenter MG, Honegger F (2000) Is lower leg proprioception essential for triggering human automatic postural responses? Exp Brain Res 130:375-391

Clair JM, Okuma Y, Misiaszek JE, Collins DF (2009) Reflex pathways connect receptors in the human lower leg to the erector spinae muscles of the lower back. Exp Brain Res 196:217-227

Cresswell AG, Oddsson L, Thorstensson (1994) The influence of sudden perturbations on trunk muscle activity and intra-abdominal pressure while standing. Exp Brain Res 98:336-341

Hodges PW (2001) Changes in motor planning of feedforward postural responses of the trunk muscles in low back pain. Exp Brain Res 141:261-266

Hodges PW, Richardson CA (1996) Inefficient muscular stabilization of the lumbar spine associated with low back pain. A motor control evaluation of transversus abdominis. Spine (Phila Pa 1976) 21:2640-2650

Hodges PW, Moseley GL, Gabrielsson A, Gandevia SC (2003) Experimental muscle pain changes feedforward postural responses of the trunk muscles. Exp Brain Res 151:262-271

Inglis JT, Horak FB, Shupert CL, Jones-Rycewicz C (1994) The importance of somatosensory information in triggering and scaling automatic postural responses in humans. Exp Brain Res 101:159-164

Jacobs JV, Horak FB (2007) Cortical control of postural responses. J Neural Transm 114:1339-1348

Jacobs JV, Henry SM, Nagle KJ (2009) People with chronic low back pain exhibit decreased variability in the timing of their anticipatory postural adjustments. Behav Neurosci 123:455-458

Jones SL, Henry SM, Raasch CC, Hitt JR, Bunn JY (2008) Responses to multi-directional surface translations involve redistribution of proximal versus distal strategies to maintain upright posture. Exp Brain Res 187:407-417

Lavender SA, Marras WS, Miller RA (1993) The development of response strategies in preparation for sudden loading to the torso. Spine (Phila Pa 1976) 18:2097-2105

Magnusson ML, Enbom H, Johansson R, Pyykko I (1990) Significance of pressor input from the human feet in anterior-posterior postural control. Acta Otolaryngol (Sotckh) 110:182-188

Magnusson ML, Aleksiev A, Wilder DG, Pope MH, Spratt K, Lee SH, Goel VK, Weinstein JN (1996) European Spine Society-the AcroMed Prize for Spinal Research 1995. Unexpected load and asymmetric posture as etiologic factors in low back pain. Eur Spine J 5:23-35

Melzack R (1975) The McGill Pain Questionnaire: major properties and scoring methods. Pain 1:277-299

Merlo A, Farina D, Merletti R (2003) A fast and reliable technique for muscle activity detection from surface EMG signals. IEEE Trans Biomed Eng 50:316-323

Moore SP, Rushmer DS, Windus SL, Nashner LM (1988) Human automatic postural responses: responses to horizontal perturbations of stance in multiple directions. Exp Brain Res 73:648-658

Radebold A, Cholewicki J, Panjabi MM, Patel TC (2000) Muscle response pattern to sudden trunk loading in healthy individuals and in patients with chronic low back pain. Spine (Phila $\mathrm{Pa} 1976)$ 25:947-954

Rushmer DS, Moore SP, Windus SL, Russell CJ (1988) Automatic postural responses in the cat: responses of hindlimb muscles to horizontal perturbations of stance in multiple directions. Exp Brain Res 71:93-102

Stokes IA, Gardner-Morse M, Henry SM, Dadger GJ (2000) Decrease in trunk muscular response to perturbation with preactivation of lumbar spinal musculature. Spine 15:1957-1964

Strutton PH, Beith ID, Theodorou S, Catley M, McGregor AH, Davey NJ (2004) Corticospinal activation of internal oblique muscles has a strong ipsilateral component and can be lateralised in man. Exp Brain Res 158:474-479

Thomas JS, Lavender SA, Corcos DM, Andersson GBJ (1998) Trunk kinematics and trunk muscle activity during a rapidly applied load. J Electromyogr Kinesiol 8:215-225

Thomas JS, France CR, Sha D, Vander Wiele N, Moenter S, Swank K (2007) The effect of chronic low back pain on trunk muscle activations in target reaching movements with various loads. Spine (Phila Pa 1976) 32:E801-E808

van Dieen JH, Selen LP, Cholewicki J (2003) Trunk muscle activation in low-back pain patients, an analysis of the literature. J Electromyogr Kinesiol 13:333-351

Zedka M, Prochazka A, Knight B, Gillard D, Gauthier M (1999) Voluntary and reflex control of human back muscles during induced pain. J Physiol 520 Pt 2:591-604 